\title{
Research on Regulator Performance Analysis and Test Method of Switching Power Supply
}

\author{
Huai-feng Wang, Ming Li, Wu-zhong Luo \\ Southwest Electronics and Telecommunication Technology Research Institute \\ Chengdu, China \\ e-mail: wanghuaifengyjs@163.com
}

\begin{abstract}
Power supply's performance is directly related to the performance of electronic devices. It puts forward higher requirements on the performance of computer switching power regulator, with widely using of high-power in family and computer overclocking technology applications. In this paper, switching power supply is modeled by using the simulink of matlab, simulations are performed to analyze the influence of source effect and loads effect on the performance of switching power regulator. Switching power regulator performance is affected both by source effect and loads effect during normal operation of computer, regulator performance under the influence of a single factor is difficult to test. For this problem, a feasible test method of switching power supply regulator performance is proposed. By building a test platform, the proposed method is verified.
\end{abstract}

Keywords-switching power supply; source effect; loads effect; regulator performance

\section{INTRODUCTION}

The power supply is an indispensable part of a variety of electronic equipment. It is widely used in a variety of terminal equipment, communication equipment and almost all electronic devices, as switching power supply having the advantages of small size, light weight and high efficiency, which is an indispensable power supply technology in electronic information industry ${ }^{[1]}$. Currently, household highpower electrical appliances are so widely used that it produces a great influence on power quality ${ }^{[2]}$, and applications of computer overclocking technology ${ }^{[3]}$ also depends on fine load capacity of the switching power supply. Therefore, it requests high performance of switching power regulator. This paper aims to research and analyze computer switching power supply's adaptability in unstable state of power grid (voltage amplitude increasing or decreasing) and transient effects of the DC output voltage under load mutation. That is to say, research on source effect and loads effect on performance of computer switching power regulator. The results of this paper will provide technical basis for testing of computer switching power supply regulator performance.

In this paper, basis components and working principle of computer switching power supply is introduced in Section II. Then, modeling via simulink package of matlab and simulations of influence of source effect and loads effect on regulator performance are performed in Section III. Regulator performance under the influence of a single factor is difficult to test. On the basis of Section II and Section III, a method for testing the regulator performance of computer switching power supply is proposed in Section IV, and the testing results are given. Finally, conclusions is concluded in Section V.

\section{COMPONENTS AND PRINCIPLE OF SWITCHING POWER SUPPLY}

There are many kinds of switching power supply. It is introduced by a series switching power supply as an example. The components of the series switching power supply are shown in Figure 1.

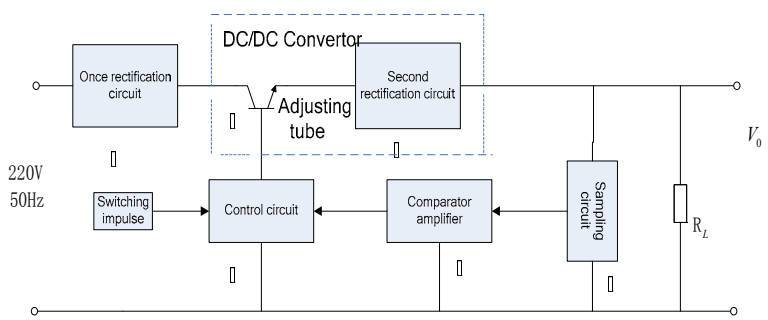

Figure 1 components of series switching power supply

It is characterized by $V_{i}$, adjusting tube $V T$, and the loads $R_{L}$ forming a series relationship. The power supply is composed of six components: (1) Once rectification and filtering circuit, $V_{i}$ is a DC voltage, denoting its output; (2) Adjusting tube, which is in switch state when the regulator circuit working, converting the input DC voltage to highfrequency pulse voltage at the output terminal, called inverter. (3) Second rectification and filtering circuit, which consists of high speed diodes, chokes and electrolytic capacitors, whose task is to converting the high-frequency pulse signal to a DC signal. (2) and (3) is named DC/DC converter, which is the core part of switching regulator power supply. Control section of the regulated power supply is composed of (4), (5), and (6), which controls the output pulse width and frequency of adjusting tube.

It has a cycle time from saturation and conduction to cutoff owing to that base input of the adjusting tube is switching pulse signal. It is assumed that $V T$ is an ideal switch, $V_{C E}=0, v_{E}=V_{i}$ when $V T$ is in the state of saturation and conduction, and $V_{C E}=\infty, v_{E}=0$ when $V T$ 
is in the state of cutoff. The output of adjusting tube's emitter is rectangular pulse, as shown in Figure 2.

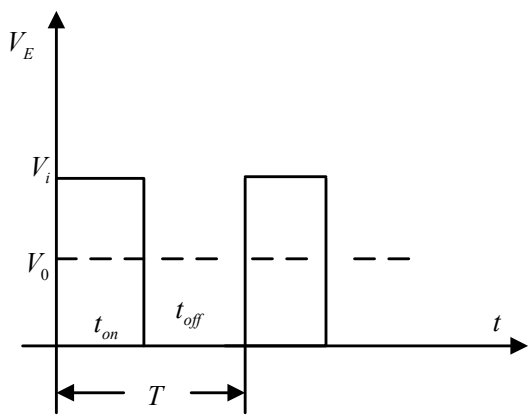

Figure 2 output rectangular pulses of adjusting tube

Average DC voltage $V_{0}$ is obtained when the rectangular pulses pass the second rectification and filtering circuit, whose amplitude is as following:

$$
V_{0}=\frac{t_{\text {on }}}{t_{\text {on }}+t_{\text {off }}} V_{i}=\frac{t_{\text {on }}}{T} V_{i}=D_{1} V_{i}
$$

$t_{\text {on }}$ and $t_{\text {off }}$ denote the saturation-conduction time and the cutoff time of $V T$ respectively. $T=t_{\text {on }}+t_{\text {off }}$ denotes the cycle time of the rectangular pulses, and $D_{1}=t_{\text {on }} / T$ denotes duty ratio of conduction time. $V_{0}$ could be controlled by $D_{1}$ when $V_{i}$ is fixed. Sampling circuit, comparator amplifier, controlling on/off time ratio circuit and adjusting tube consist of a negative feedback loop. The output rectangular pulses of adjusting tube become narrow controlled by the negative feedback loop, when $V_{0}$ increases. $t_{o n}$ decreasing leads to $D_{1}$ decreasing, then the increasing of $V_{0}$ is limited. On the contrary, $D_{1}$ increasing limits $V_{0}$ decreasing, controlled by the negative feedback loop, when $V_{0}$ decreases. Thus it can be seen that output voltage $V_{0}$ is kept stable.

\section{SimulinK MOdeling fOR SOURCE EFFECT AND LOADS EFFECT}

It is difficult to create a uniform transfer function due to that switching power supply is a discrete and nonlinear system. Simulink is a system package, using for modeling, simulating and dynamic analysis, which runs under MATLAB for modeling. It is an important step in the process of switching power supply design, which using power system toolbox in simulink package to analyze switching power supply ${ }^{[4-10]}$.

- Modeling of input circuit

It is easy to establish the input circuit simulation model, using the power module and the resistor-capacitor module of the electric system toolbox, as shown in Figure 3.

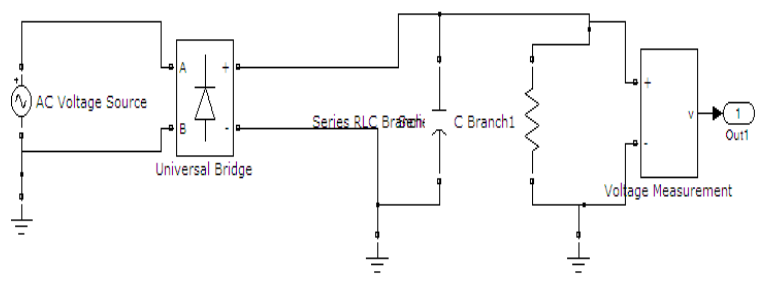

Figure 3 modeling of input circuit

- Modeling of DC/DC converter

Its circuit is shown in Figure 4.

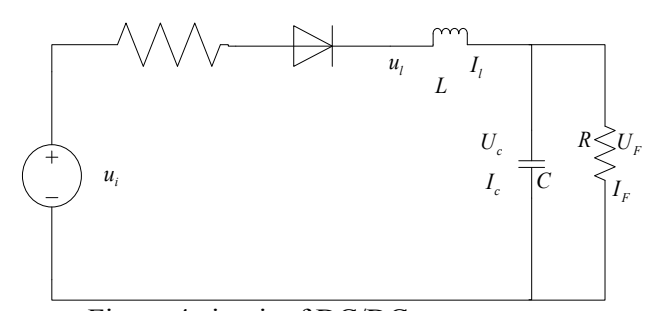

Figure 4 circuit of DC/DC converter

It needs to establish a non-linear model, since that current could not pass the rectifier diodes inversely, as illustrated in Figure 4. Current in inductor is as following:

$$
I_{L}=\frac{1}{L} \int U_{L} d t=\frac{1}{L} \int\left(U_{i}-U_{F}\right) d t
$$

$U_{i}$ and $U_{L}$ denote output voltage of rectification circuit and voltage of inductor, respectively. $U_{F}$ denotes load voltage, which is as following:

$$
U_{F}=U_{C}=\frac{1}{C} \int I_{C} d t=\frac{1}{C} \int\left(I_{L}-I_{F}\right) d t
$$

$U_{C}, I_{L}, I_{C}$ and $I_{F}$ denote capacitor voltage, inductor current, capacitor current and load current, respectively. Model of DC/DC converter is created by simulink on the basis of above analysis, as shown in Figure 5.

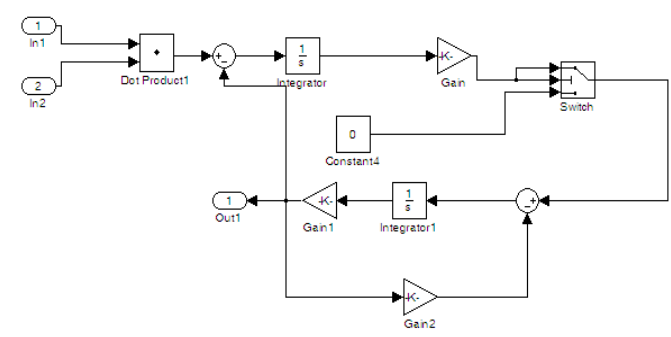

Figure 5 modeling of DC/DC converter

- $\quad$ Modeling of PWM controller

The simulation model uses pulse generator in simulink package to create triangle wave, basing on the integral relationship, whose frequency, amplitude and duty ratio are fixed. Pulse width modulation (PWM) model simulation module is shown in Figure 6. 


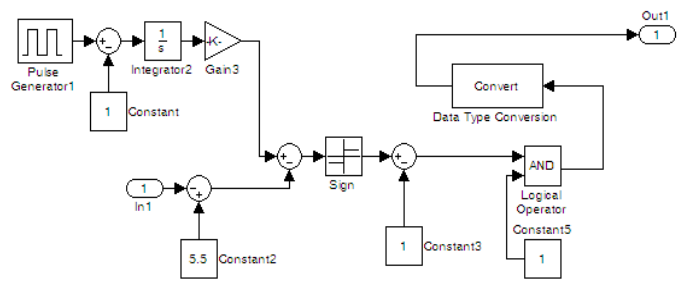

Figure 6 modeling of PWM

The above three parts are linked as shown in Figure 7.

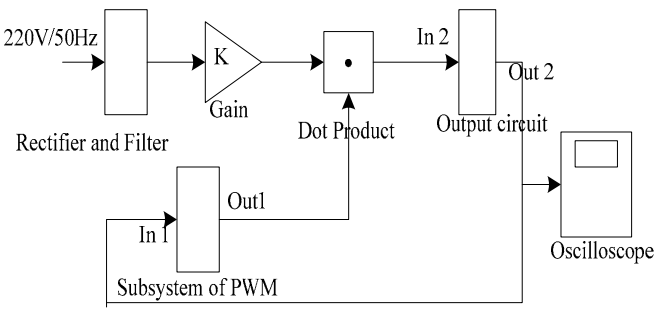

Figure 7 modeling of switching power supply

A complete switching power supply simulation model is established after setting the parameters of each device according to the actual circuit. The simulation results of output DC under 5 volt of terminal is shown in Figure 8.

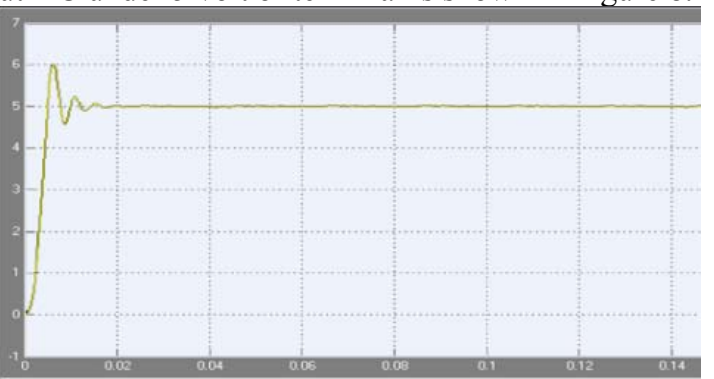

Figure 8 results of output DC under 5 volt of terminal

\section{A. Source Effect}

Source effect refers to the effect that only the change of input leads to the change of output. Switching power supply model is improved by establishing M-file, which makes the output of the AC voltage increasing fluctuations and keeps the load constant. The output DC voltage increases by about 0.215 volt, as shown in Figure 9.

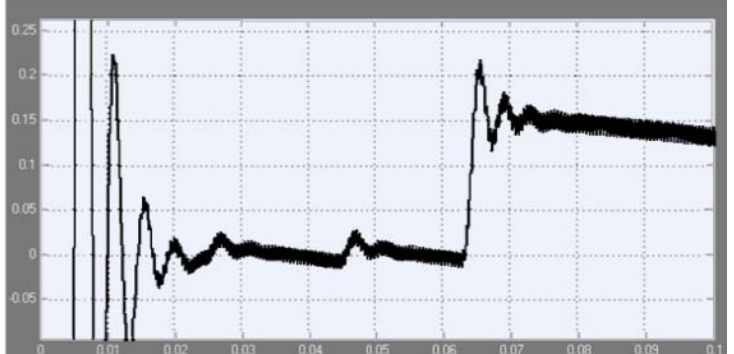

Figure 9 waveform of output DC voltage caused by source effect

\section{B. Loads Effect}

Loads effect refers to the effect that only the change of load leads to the change of output. The waveform of the output DC voltage fluctuates causing by instantaneous changes in load value in the process of simulation, as shown in Figure 10.

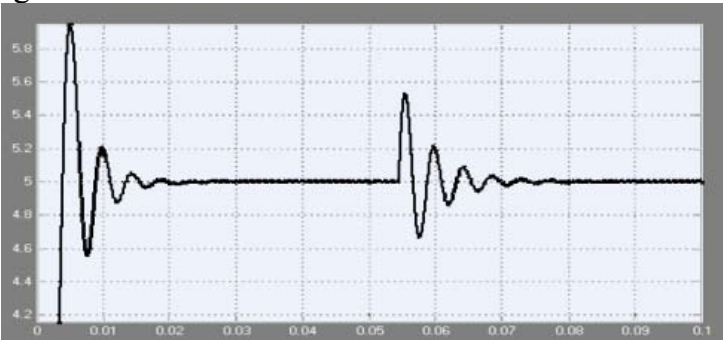

Figure 10 waveform of the output DC voltage caused by loads effect

The source effect of switching power supply causes output DC voltage instability, which leads to the computer running error, unable to work, or even burned, when the regulator performance is poor. The computer can only work properly under light loads, and it works abnormally under high loads, if the power load capacity is poor. Actual test of regulator performance will be performed using the proposed method in Section IV.

\section{Test Method And Regulator PeRformance ANALYSIS}

Switching power supply's regulator performance is affected both by source effect and loads effect during normal operation of computer. Regulator performance under the influence of a single factor is difficult to test. For this problem, a feasible test method of switching power supply regulator performance is proposed. Testing for regulator performance is performed basing on the proposed method.

\section{A. Source Effect on Regulator Performance of Switching Power Supply}

- Test equipment: a precision purifying $\mathrm{AC}$ power supply (YaGuang-JJW2-5KVA), an AC voltage regulator, a digital oscilloscope (Tektronix DPO 7254), an ordinary desktop computer.

- Test environment

Test environment of source effect is built as shown in Figure 11.

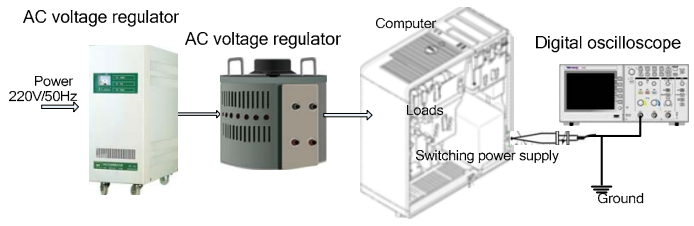

Figure 11 test environment of source effect

- Test method

In order to eliminate loads effect of switching power supply's output voltage and the influence to switching power supply caused by unload, desktop computers works in stable state and do not run any software. AC power supply is used to eliminate the impact on the test results from poor grid quality, and $\mathrm{AC}$ voltage regulator is used to modulate the switching power supply AC input voltage. The voltage 
amplitude adjustment ranges from 90 volt to 240 volt. Connect DC output terminal of switching power supply to digital oscilloscope with P6139 voltage probe. The real-time dynamic change of DC output voltage is monitored in order to test the source effect.

- Results of the test

DC output voltage of switching power supply is kept stable and the computer works properly when amplitude of $\mathrm{AC}$ input voltage ranges from 100 volt to 240 volt. Computer will be automatically closed down if the input voltage is too low, when the amplitude of AC input voltage is less than 100 volt. The source effect of DC output voltage is shown in Figure 12.

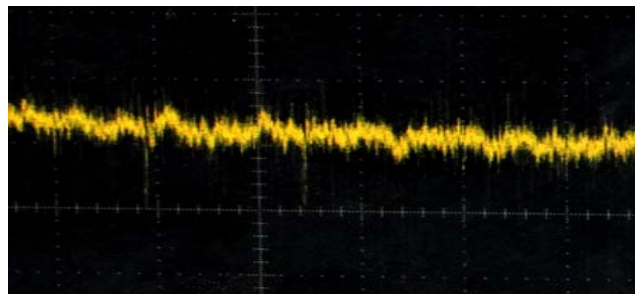

Figure 错误!未找到引用源。 source effect of DC output voltage

The test results show that source effect impacts on switching power supply lightly. The regulator performance of the tested computer's switching power supply is superior when the input $\mathrm{AC}$ voltage ranges normally.

\section{B. Loads Effect on Regulator Performance of Switching Power Supply}

- Test equipment: a precision purifying $\mathrm{AC}$ power supply (YaGuang-JJW2-5KVA), a digital oscilloscope (Tektronix DPO 7254), an ordinary desktop computer, a multi-meter.

- Test environment

In order to test the loads effect on the regulator performance of computer switching power supply, test environment is built as shown in Figure 1. CPU Power Regulation Software is developed to simulate the changes of computer loads accurately.

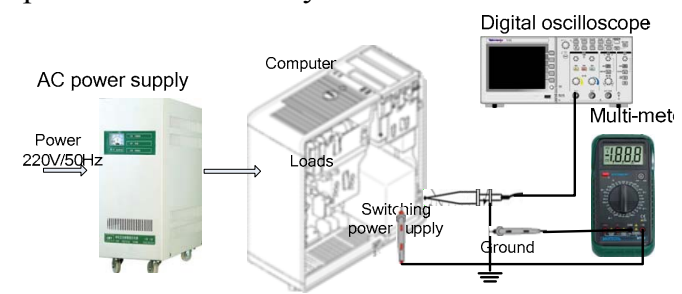

Figure 12 test environment of loads effect

Principle of the CPU Power Regulation Software: CPU occupancy rate refers to the ratio of total time of CPU busy (running applications) and the time of refresh cycle. The computer's task manager shows the statistical average of usage in each refresh cycle. The change of CPU power is decided by the change of occupancy rate, which is the change of loads in reality. Sinusoidal waveform and straight line waveform of fixed occupancy is drawn by task manager using CPU Power Regulation Software, as shown in Figure 14 and Figure 15.

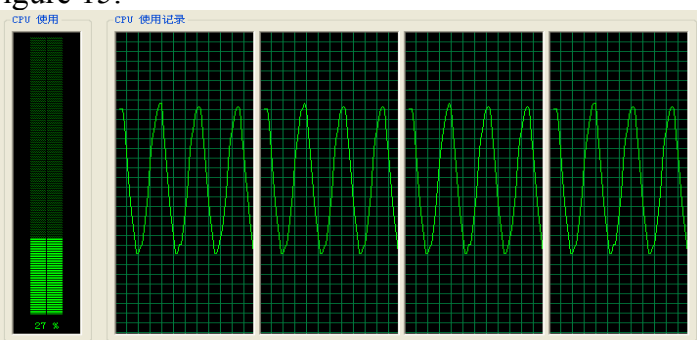

Figure 13 sinusoidal waveform draw with CPU Power Regulation Software

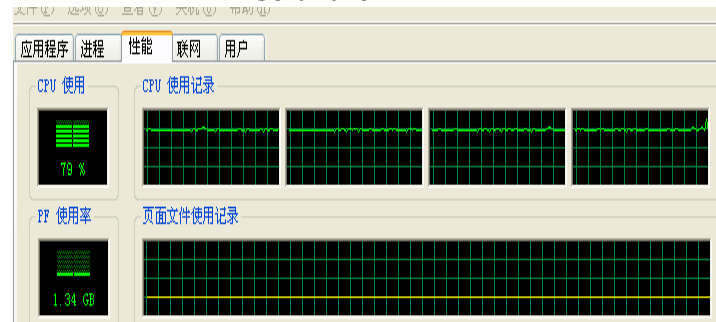

Figure 14 straight line waveform draw with CPU Power Regulation Software

- $\quad$ Test Method and Results

The change of computer loads is simulated by CPU occupancy rate adjustment method. Monitor influences to DC output voltage by instantaneous change of loads, using digital oscilloscope. The test results are shown in Figure 16

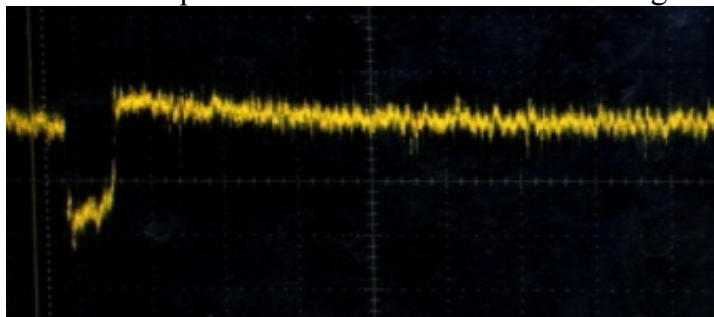

Figure 15 influence of instantaneous change of loads on the DC voltage

As illustrated in Figure 16, DC output voltage changes by about 100 millivolt under the influence of loads effect and returns to regulation state within a very short time.

Values of DC output current are tested by multi-meter under different loads, as illustrated in Table 1. Output DC voltage decreases with the loads increasing, which is owing to internal resistance of the computer switching power supply.

\begin{tabular}{|c|c|}
\hline Table 1 Test results of 12 volt output terminal \\
\hline CPU Occupancy Rate & $\begin{array}{c}\text { Voltage of 12 volt Output } \\
\text { Terminal(volt) }\end{array}$ \\
\hline $10 \%$ & 11.56 \\
\hline $30 \%$ & 11.53 \\
\hline $50 \%$ & 11.50 \\
\hline $80 \%$ & 11.47 \\
\hline $100 \%$ & 11.46 \\
\hline
\end{tabular}


The regulator performance of the computer switching power supply in the testing environment is superior under the influence of source effect and loads effect, and regulator performance of source effect is better than the one of loads effect, as depicted in Figure 12, Figure 16 and Table 1.

\section{CONCLUSION}

It is shown that regulator performance of switching power supply is affected both by source effect and loads effect during normal operation of computer by modeling and simulating. A test method under the influence of a single factor is proposed. With building the test platform, the proposed method in this paper is proved. The results can provide a theoretical basis for testing of computer switching power regulator performance.

\section{REFERENCES}

[1] S. Hashimoto,S.NaKa,K.Toya and U. Sosorhang. "Development of Intelligent Switching Power Supply Based on Advanced Digital Control". 2010 5th IEEE conference on Industrial Electronics and Applications.

[2] Zhu Guiping,Wang Shumin.A survery on power quality control technology[J];Automation of electric power systems;2002-19.
[3] Ahmed, Hikmat and Azween, Abdullah (2009) The Impact of Overclocking the CPU to the Genetic Algorithm. international journal of computer science and network security, 9 (5). pp. 175-180.

[4] D. Hanselman, B.Littlefield .Mastering matlab 6 a comprehensive tutorial and reference[M].

[5] Zhang Jia-sheng, Zhang Lei.Researh on an general unified modeling and analysis approach of 4-quadran converters $[\mathrm{J}]$. Processing of the CSEE,2004,24(8):39-44.

[6] Lai Xinquan, Zhou Lixia,Chen Fuji.Design of a dynamic slope compensation circuit for boost DC-DC converter[J]. Microelectronics, 2005,35(4):421-423.

[7] Cuinjoan F,Calente J,Poveda A,et al.Large Signal Modeling and Simulation of Switching DC-DC Converters[J]. IEEE Trans Electronics, 1997,12(3):485-494.

[8] A.M. Wu, J.Xiao , D. Markovic, S.R. Sanders."Digital PWM Control: Aapplication in Voltage Regulator Models," IEEE Power Electronics Specialists Conference,1997 Record,pp.77-83.

[9] A.Prodic and D,Maksimovic,"Digital PWM Controller and Current Estimator for a Low-Power Switching Converter," IEEE Computers in Power Electronics 2000,pp.123-128.

[10] Y.W. Martin and S.S. Ang, "Digital Control of Switching Converters," IEEE Symposium on Industrial Electronics, vol.2,20025,pp.480-484. 